\title{
A common fixed point for operators in probabilistic normed spaces
}

\author{
M.B. Ghaemi ${ }^{a, *}$, Bernardo Lafuerza-Guillen ${ }^{b}$, A. Razani ${ }^{\text {c }}$ \\ "Factily of Mathematics, Iran Unitersity of Science and Techmology. Narmak, Tethon. Iran \\ bepartment of Applied Mathomatics. Unitersity of Almeria. Almeria. Spaü \\ 'Deparment of Mathematics, Fuculty of Science, I. Kh. International Unitersity, P.O. Box 34194-288, Quzkin, Iran
}

Accepted 7 September 2007

\begin{abstract}
Probabilistic Metric spaces was introduced by Karl Menger. Alsina, Schweizer and Sklar gave a general definition of probabilistic normed space based on the definition of Menger (Alsina C, Schweizer B, Sklar A On the definition of a probabilistic normed spaces. Aequationes Math 1993,46:91-8] Here, we consider the equicontinuity of a class of linear operators in probabilistic normed spaces and finally, a common fixed point theorem is proved. Application to quantum Mechanic is considered.
\end{abstract}

Q 2007 Elsevier Ltd. Ail rights reserved.

\section{Introduction}

The theory of probabilistic metric spaces, introduced in 1942 by Meneger [10], was developed by numerous authors, for instance [4], as well as those in $[12,13]$. The notion of a probabilistic metric space corresponds to the situations when we do not know exactly the distance between two points, we know only probabilities of possible values of this distance. Such a probabilistic generalization of metric spaces appears to be well adapted for the investigation of quantum physics as shown by El Naschic. The notion of a probabilistic normed space was introduced by Serstrev [14]. Alsina, Schweizer and Sklar gave a general definition of probabilistic normed space based on the definition of Menger [13] for probabilistic metric spaces in $[3,2]$. Linear operator in probabilistic normed spaces has been studied by Guillen, Lallena and Sempi in $[8,9]$. In this paper, we consider the equicontinuity of a class of linear operators on probabilistic normed space and by applying these results, a common fixed point theorem is proved. In order to do this, we recall some definitions from $[1-3,6]$.

Definition 1.1. A distribution function is a function $F: \overline{\mathrm{R}} \rightarrow(0,1)$ that is nundecreasing and left continuous on $\mathrm{R}$; moreover, $f-\infty)=0$ and $F(\infty)=1$.

\footnotetext{
- Corresponding author.

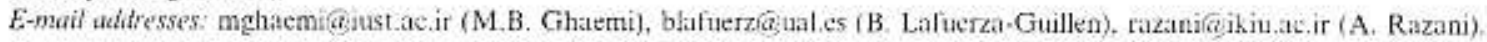


The set of all the distribution functions is denoted by $\Delta$ and the set of those distribution functions such that $F(0)=0$ is denoted by $\Delta^{+}$. The distance distribution functions are denoted by $D^{+}$and $D^{+}=\left\{F \in \Delta^{+} ; \lim _{x \rightarrow \infty} F(x)=1\right\}$. A natural ordering in $\Delta$ and $\Delta^{+}$is defined by $F \leqslant G$ whenever $F(x) \leqslant G(x)$ for every $x \in R$. The maximal element in this order for $A^{+}$is $\epsilon_{1}$, where for $a \leqslant \infty$ the distribution function $\epsilon_{d}$ is defined by

$$
\epsilon_{i}= \begin{cases}0, & \text { if } t \leqslant a, \\ 1, & \text { if } t>a\end{cases}
$$

Definition 1.2. A triangle function is a binary operation on $A^{+}$that is tommutative, associative, nondecreasing in each place, and has $\varepsilon_{0}$ as identity. $4^{+}$.

Note that the continuity of a triangle function means continuity with respect to the topology of weak convergence in

Example 1.3. Let $T$ be a continuous $t$-norm, i.e. a continuous binary operation in $[0,1]$ that is associative, nondecreasing and has 1 as identity; $T^{*}$ is a continuous $t$-conorm, namely a continuous binary operation on $[0,1]$ that is related to a continuous s-norm through

$$
T^{*}(x, y)=1-T(1-x, 1-y) \text {. }
$$

Typical continuous triangle functions are convolution, the operations $\tau_{T}$ and $\tau_{r}$, which are given by

$$
\tau_{r}(F, G)(x)=\sup _{x+1=x} T(F(s), G(t))
$$

and

$$
{ }_{i} r(F, G)(x)=\inf _{x \rightarrow t \in m} T^{*}(F(s), G(t))
$$

for all $F, G$ in $A^{+}$and all $x$ in $P[13$, Sections 7.2 and 7.3], respectively.

It follows without difficulty from the above that:

$$
\tau_{T}\left(\varepsilon_{n}, \varepsilon_{b}\right)=\hat{c}_{i+b}=\tau_{r}-\left(\varepsilon_{a}, \hat{\varepsilon}_{b}\right)
$$

for any continuous $t$-norm $T$, any continuous $t$-conorm $T^{*}$ and any $a, b \geqslant 0$.

Definition 1.4. A probabilistic normed space (briefly, $\mathrm{PN}$-space) is a quadruple $\left(X, \Theta, \tau, \tau^{*}\right)$, where $X$ is a real vector space, $\tau$ and $\tau^{*}$ are continuous triangle functions, and $\Theta$ is a mapping from $X$ into $A^{+}$such that, for $p, q \in X$, the following conditions hold:

(PNI) $\Theta_{p}=\epsilon_{0}$ iff $p=0$ where 0 is the null vector in $X$,

(PN2) $\Theta_{-r}=\Theta_{\rho}$

(PN3) $\Theta_{p+q} \geqslant \tau\left(\Theta_{\rho}, \Theta_{i}\right)$.

(PN4) $\Theta_{p} \leqslant \tau^{*}\left(\Theta_{x p}, \Theta_{1-x ; p}\right)$, for all $z \in I=[0,1]$

If $(X,\|-\|)$ is a real normed space, $\tau$ a triangle function such that $z\left(\epsilon_{a,}, \epsilon_{b}\right) \leqslant \epsilon_{i r}+b$ for all $a, b \geqslant 0$, and if $\Theta: X \rightarrow \Delta^{\prime}$ is defined via $\Theta_{\mu}=\epsilon_{|p|}$, then $\left(X, 0, \tau, \tau^{*}\right)$ is a PN-space.

Note that if $\tau^{*}=\tau_{M}$ (where $\tau_{M}$ is the $t$-norm defined as $\tau_{M}(x, y)=\operatorname{Min}\{x, y)$ ) and equality holds in (PN4), then $(S, \Theta, \tau, \tau M)$ is a Serstnev PN-space [1]. In this case, as shown in [3], the conditions

$$
\Theta_{\mu}=\tau_{u}\left(\Theta_{z \mu}, \Theta_{(1-x \mid \mu)}\right) \text { for all } p \text { in } X \text { and } \alpha \text { in } I
$$

and (PN2), taken together, are equivalent to Serstnev's condition

$$
\Theta_{i p}(x)=\Theta_{\mu}\left(\frac{x}{|i|}\right) \text { for all } i \in \mathbb{R}-\{0\} \text { and } x \text { in } \mathbb{R} \text {. }
$$

Every PN-space $\left(V, \Theta, \tau, \tau^{*}\right)$ can be endowed with the strong topology; this topology is generated by the strong neighborhood, which are defined as follows: for every $t>0$, the ncighborhood $N_{p}(t)$ at a point $p$ of $V$ is defined by

$$
N_{p}(t):=\left\{q \in V: d_{S}\left(\Theta_{p-q}, \varepsilon_{0}\right)<t\right\}=\left\{q \in V: \Theta_{p-q}(t)>1-t\right\}
$$


Definition 1.5. A topological vector space (TVS) is a vector space $V$ together with a topology such that with respect to this topology:

(i) The map of $V \times V-V$ defined by $(x, Y) \mapsto x+y$ is continuous.

(ii) The map of $\mathrm{F} x X \rightarrow X$ defined by $(\alpha, x) \rightarrow x x$ is continuous, Where $\mathrm{F}$ is $\mathrm{AR}$ or $\mathrm{C}$.

Definition 1.6. A topological vector space $X$ is called locally convex if the neighborhood filter around 0 has a basis of convex sets.

Note that every PN-space $\left(\nu, \Theta, \tau, \tau^{*}\right)$, when it is endowed with the strong topology induced by the probabilistic norm $\Theta$ is a topological vector space if, and only if, for every $p \in V$ the map from R into $V$ defined by $\alpha \rightarrow \alpha p$ is continuous (see [2]) for more details). Henceforth a PN-space $\left(V, \Theta, \tau, \tau^{*}\right)$ which is a topological vector space is denoted by TV PN-space.

Remark 1.7. It was proved [3, Theorem 4] that if the triangle function $\tau^{*}$ is Archimedean, i.e. if $\tau^{*}$ admits no idempotents other than $\hat{c}_{0}$ and $f_{(x)}[13]$ then the mapping $\alpha \mapsto \alpha p$ is continuous and as a consequence of this, the PNspace $\left(V, v, \tau, \tau^{*}\right)$ is a TV PN-space.

Definition 1.8. A PN-space $\left(V, v, \tau, \tau^{*}\right)$ is characteristic if $v(V) \subseteq \mathscr{Y}^{+}$, or equivalently $v_{p} \in \mathscr{Y}^{+}$for every $p \in V$.

Theurem 1.9. A characteristic Šerstnev space $(V, v, \tau)$ with $\tau=\tau_{M}$ is locally contcx.

Proof. ([11]). We prove it here because the notation in Prochaska's thesis is different from the one that has become usual after the publication of [12].

It suffices to consider the family of neighborhoods of the origin $\theta, N_{0}(t)$, with $t>0$. Let $1>0, p, \eta \in N_{0}(t)$ and $\alpha \in[0,1]$. Then

$$
\begin{aligned}
& v_{z p+(1-z i q}(t) \geqslant \tau_{M}\left(v_{x p}, v_{(1-\gamma \mid q)}\right)(t)=\sup M\left(v_{x p}(\beta t), v_{i 1-x_{i q}}((1-\beta) t): \beta \in[0,1]\right) \geqslant M\left(v_{\alpha p}(\alpha t), v_{(1-x i q}((1-\alpha) t)\right) \\
& =M\left(v_{p}(t), v_{q}(t)\right)>1-t .
\end{aligned}
$$

Thus $\alpha p+(1-\alpha)_{q}$ belongs to $N_{\theta}(t)$ for every $\alpha \in[0,1]$.

Theorem 1.10. Let $\left(V, v, \tau, \tau^{*}\right)$ be a $T V P_{N}$-space. If $A: X \rightarrow X$ is linear and contimusus at 0 , then $A$ is contimuots.

A linear operator $T: V_{1} \rightarrow V_{2}$ where $\left(V_{1}, v_{1}, \tau_{1}, z_{i}\right)$ and $\left(V_{2}, V_{2}, \tau_{2}, \tau_{2}^{*}\right)$ are TV PN-spaces, is bounded if it transform bounded subset of $V_{1}$ into bounded subset of $V_{2}$. Note that continuous linear operators are bounded.

Remark 1.11. A linear operator between two lotally convex TV PN-spaces is continuous if and only if it is bounded, see $[5$, p. 477].

\section{Common fixed point}

In this section, we prove some theorems and as a result of these theorems, one can prove the existence of a common fixed point theorem. Due to this, the next result is a uniform boundedness theorem for TV-PN spaces.

Theorem 2.1. If $T$ is a collection of contimuons linear maps betweon $t_{1}$ TV $P N$-spacis $\left(V_{1}, V_{1}, \tau_{1}, \tau_{1}^{*}\right)$ and $\left(V_{2}, V_{2}, \tau_{2}, \tau_{2}^{*}\right)$ and if the set

$$
\Gamma(x)=\{\Lambda x: A \in \Gamma\}
$$

is a bounded subset of $V_{2}$ for ctery $x \in V_{1}$, then $\Gamma$ is equicontintous.

Proof. Let $N_{\mathrm{u}}(t)=\left\{p \in V_{1}: v_{p}(t)>1-1\right\}$ be a neighborhood of 0 , then

$$
\overline{N_{01}\left(\frac{1}{3} t\right)}=\overline{\left\{p: v_{p}(t)>1-\frac{t}{3}\right\}}=\left\{p \in V_{1}: d_{s}\left(t_{\rho}, \varepsilon_{01}\right) \leqslant \frac{1}{3}\right\}
$$


and we have

$$
\overline{N_{0}\left(\frac{1}{3} t\right)}+\overline{N_{0}\left(\frac{1}{3} t\right)} \subset\left\{p \in V_{1}: d_{s}\left(v_{j}, z_{0}\right) \leqslant 1\right\} .
$$

Put

$$
E=\bigcap_{A \in r^{-1}}\left(N_{0}\left(\frac{1}{3} t\right)\right) .
$$

$V_{1}$ is a complete metric space and $V_{1} \subset U_{n=1}^{\infty} n E$. Therefore $E$ is a closed subset of $V_{1}$ also the interior of $E$ is not empty. Hence $x-E$ contains a neighborhood $N_{b}(s)$ of 0 such that

$$
A\left(N_{0}(s)\right) \subset A(x)-A(E) \subset \overline{N_{0}\left(\frac{1}{3} t\right)}+\overline{N_{0}\left(\frac{1}{3} i\right)}
$$

for every $A \in I$. This proves $\Gamma$ is equicontinuous.

Corollary 2.2. If $T$ is a collection of continutas linear maps from PN-space $\left(V_{1}, \tau_{1}, \tau_{1}, \tau_{1}\right)$ onto PN-space $\left(V_{2}, v_{2}, \tau_{2}, \tau_{2}\right)$. where $\tau_{1}^{*}$ and $\tau_{2}^{+}$are Archinedean and $\Gamma(x)=\{A x ; A \in \Gamma)$ is a bounded subset of $V_{2}$ for twery $x \in V_{1}$, then $\Gamma$ is equicontintous.

Proof. By Remark 1.7 the PN-spaces $\left(V_{1}, v_{1}, \tau_{1}, \tau_{j}\right)$ and $\left(V_{2}, r_{2}, \tau_{2}, \tau_{2}^{*}\right)$ are TV PN-spaces, Now the result follows from Theorem 2.1.

Corollary 2.3. If $\Gamma=\left\{A: V_{1} \rightarrow V_{2}\right\}$ is a collection of continnons linear maps, where $\left(V_{1}, V_{3}, \tau_{1}, \tau_{1}^{*}\right)$ ant $l\left(V_{2}, v_{2}, \tau_{2}, \tau_{2}^{*}\right)$ are characteristic Serstne spaces with $\tau_{1}=\tau_{2}=\tau_{M}$ and if $\Gamma(x)=\{A x ; A \in I\}$ is a bounded subset of $V_{2}$, for esery $x \in V_{1}$, then $\Gamma$ is equicontinuous.

Proof. Note that $V_{1}$ and $V_{2}$ are locally convex spaces by [8. Theorem 7]. Now the result is immediate conseguence of Theorem 2.1.

Theorem 2.4. If $\left\{A_{n}\right\}$ is a secpuence of continuous linear mapping from a $T V P N$-space $\left(V_{1}, v_{1}, \tau_{1}, \tau_{i}\right)$ into a $T V$ PN-space $\left(V_{2}, v_{2}, \tau_{2}, \tau_{2}^{*}\right)$ and if $A x=\lim _{n \rightarrow \infty} A_{i} x$, exist for exery $x \in V_{1}$, then $A$ is contintous.

Provf. Theorem 2.1 implies that $\left\{A_{n}\right\}$ is equicontinutous. Suppose $U_{2}$ is a neighborhood of 0 in $V_{2}$, ther $A_{n}\left(U_{1}\right)=U_{2}$ for all $n \in \mathbb{N}$ and some neighborhood $U_{1}$ of 0 in $V_{1}$. It follows that $A\left(U_{1}\right) \subseteq \overline{V_{2}}$, hence $A$ is continuous.

Definition 2.5. A linear operator $T$ of $V_{1}$ into $V_{2}$ is called bounded if it transform bounded subset of $V_{1}$ into bounded subset of $V_{2}[5$, p. 63].

Corollary 2.6. If $\left\{A_{a}\right)$ is a sequence of hounded linear mapping from a characteristic Scrstnet $\left(V_{1}, v_{1}, \tau_{1}, \tau_{1}\right)$ into a characteristic Serstnen $\left(V_{2}, v_{2}, \tau_{2}, \tau_{2}^{*}\right)$ with $\tau_{1}=\tau_{2}=\tau_{M}$ and if $A x=\lim _{n \rightarrow \infty} A_{n} x$, exist for every $x \in V_{1}$, then $A$ is bounded.

Proof. By Remark $1,11, \lambda_{n}$ is continuous for all $n$. Now the result is a immediate consequence of Theorem 2,4 .

Lemma 2.7. In a characteristic Serstnev $\left(V, v, \tau, \tau^{*}\right)$ the following statement are equizatent for a subset $A$ of $V$

$A$ is $\mathscr{Q}$-bounded

$A$ is topologicaly bounded

Proof. (a) $\Rightarrow$ (b) Let $A$ any $\mathscr{L}$-bounded subset of $V$ and let $p_{n}$, be any sequence of elements of $A$ and $\alpha_{n}$ any sequence of real numbers that converges to 0 ; there is no loss of generality in assuming $\alpha_{n}=0$ for every $n \in \mathbb{N}$

$$
v_{n_{i, p_{n}}}(x)=v_{p_{n}}\left(\frac{x}{\left|\alpha_{i}\right|}\right) \geqslant R_{i}\left(\frac{x}{\left|\alpha_{n}\right|}\right) \rightarrow 1
$$

as $n \rightarrow+\infty$. 
Thus $\alpha_{n} p_{n} \rightarrow Q$ in the strong topology and $A$ is topologically bounded. (b) $\Rightarrow$ (a) Let $A$ be a subset of $V$ which is not S-bounded. Then

$$
R_{A}(x) \rightarrow y<1
$$

as $x \rightarrow+\infty$.

By definition of $R_{A}$ for every $n \in \mathbb{N}$ ther is $p_{n} \in A$ such that

$$
v_{p_{n}}\left(n^{2}\right)<\frac{1+\gamma}{2}<1 \text {. }
$$

If $\alpha_{n}=1 / n$, then

$$
v_{s_{n}, p_{4}}(1 / 2) \leqslant v_{3_{n} p_{n}}(n)=v_{p_{n}}\left(n^{2}\right)<\frac{1+\eta}{2}<1 .
$$

which shows that $v_{x_{,}, p_{0}}$ does not tend to $\varepsilon_{0}$, even if it has a weak limit, viz, $x_{n} p_{n}$ does not tend to $\theta$ in the strong topology; in other words, $A$ is not topologically bounded.

The following corollary is immediate from Corollary 2.6 and Lemma 2.7.

Corollary 2.8. If $\Gamma=\left\{A: V_{1} \rightarrow V_{2}\right\}$ is a collection of contimesrs lintar maps, where $\left(V_{1}, V_{1}, \tau_{1}, \tau_{1}^{*}\right)$ and $\left(V_{2}, V_{2}, \tau_{2}, \tau_{2}^{*}\right)$ are characteristic Serstnev spaces wish $\tau_{1}=\tau_{2}=\tau_{1}$ and if $\Gamma(x)=[A x, A \in \Gamma]$ is a D-bouanded suhset of $V_{2}$ for every $x \in V_{1}$, then $\Gamma$ is equicontimous.

In order to give the final result of this article, the following definition is given:

Definition 2.9. A set $A \subset V$ is balanced, if $\alpha x \in A$ whenever $x \in V$ and $|x| \leqslant 1$. A set $A$ is absorbing, if for each $x \in V$ there is an $\varepsilon>0$, such that $t x \in A$ for $0<1<\varepsilon$.

Theorem 2.10. Suppose $K$ is a nonempty convex compact subset of locally contex $T V P N-s p a c e\left(V, 1, \tau, \tau^{*}\right)$. Let $G$ he a group of linear mapping such that

$$
\Gamma(x)=\{\Lambda x: A \in G\}
$$

is bounded in $V$ for etery $x \in V$. Afso $A(K) \subseteq K$ for every $A \in G$. Then $G$ has a common fixed point in $K$ : that is there exist $p \in K$ such that $A p=p$ for every $A \in G$.

Proof. The group $G$ is equicontinuous by Theorem 2.1 and $V_{1}$ has a local base consisting of balanced convex set $U$ which satisfies $A(U) \subseteq U$ for every $A \subseteq G$. Let $\Omega$ be the collection of all nonempty convex sets $H \subseteq K$ such that $A(I)=U$ for every $A \in G$. Now, we partially ordered the set $\Omega$ by set inclusion. Then Hausdorft's maximality theorem shows that $\Omega$ contains a maximal totally ordered subcollection $\Omega_{0}$. The intersection $H_{0}$ of all members of $\Omega_{0}$ is a minimal member of $\Omega$. Now, it is enough to show that $H_{0}$ have exactly one point. Otherwise, if $H \in \Omega$ contains more than one point. Then $H-H \neq\{0\}$ and there is a convex balanced member of the above local base which does not cover $H-H$. Since $H-H$ is compact, there exists some $s>0$ such that $H-H \subset s U$. Let $r$ be the greatest lower bound of these member 3 . Set $W=t U$, then $W$ is a convex balanced open set such that

$$
(1-r) \bar{W} \text { does not cover } H-H \text { il } 0<r<1 \text {. }
$$

$I I$ is compact and hence, there exist $x_{1}, x_{2}, \ldots, x_{n} \in H$ such that $H \subset \bigcup_{i=1}^{n}\left(x_{i}+1 / 2 W\right)$. Let $r=1 / 4 n$ and define $H_{1}=H \bigcap_{\text {we }}(y+(1-r) \bar{W}) \cdot H_{1}$ is compact, convex and $A H_{1} \subset H_{1}$ for every $A \in G$. By (1) there are points $x \in H$ and $y \in H$ such that $x-y$ does not lie in $(1-r) \bar{W}$. Any such $x$ is not in $H_{1}$. Thus $H_{1} \neq H$. The point $x_{0}=1 / m$; $\left(x_{1}+x_{2}+\cdots+x_{n}\right) \in H_{1}$ and therefore $H_{1} \neq \Phi$. This shows that $H_{0}$ contains only one point.

The following corollaries are immediate consequence from Theorems 2.10 and 1.9 .

Corollary 2.11. Suppose $K$ is a nonempty convex sabset of characteristic Serstnet space $\left(V, v, \tau, \tau^{*}\right)$ with $\tau=\tau, M_{\text {. Let }} G$ be a group of linear mapping such that $\Gamma(x)=\{A x: A \in G\}$ is bounded in V for every $x \in V$. Morever, $A(K) \subseteq K$ for every $A \in G$. Then $G$ has a common fixed point in $K$

Corollary 2.12. Suppose $K$ is a nonemply comes subset of charateristic Serstwet space $\left(V, v^{\prime}, \tau, \tau^{*}\right)$ with $\tau=\tau_{M}$ and $A$ is an invertible operator on $V$ such that the set $\left\{A x, A^{-1} x\right\}$ is bounded for tery $x \in V$, then $A$ and $A^{-1}$ has a common fixed poins 


\section{Application in plivsics}

Menger sponge is a random space which could be used for instance to predict the Background micro wave radiation (see El Naschie and also He's Book [7]).

\section{Conclusions}

In this worked we have analyzed in sone detail the problem of equicontinuity of a class of linear operators on probabilistic normed spaces. We have shown for the class of characteristic Serstnev spaces if $\Gamma(x)=\{A x, A \in \Gamma\}$ is bounded then $\Gamma$ is equicontinuous. $A$ detailed study of how we can have a common fixed point for a group of linear operators is given.

\section{References}

[1] Alsina C, Schweizer B. Sempi C, Sklar A. On the definition of a probabilistic inner product space. Rend Mat Appl 1997:17:115-27.

[2] Alsina C, Schweizer B, Sklar A. Continuity properties of probablistic norms. J Math Anal Appl 1497;208-446-52.

[3] Alsina C, Schwejzer B, Sklar A. On the definition of a probabilistic normed spaczs. Aequationes Math 1993:46:91 - 8.

[4] Constantin G, Istrateseu 1. Elements of probablistic analysis. Kluwer Academic Publishers.; 1989.

[5] Edwards RE. Functional analysis theory and application. Dover; 1995.

[6] Ghaemi MB. Razani A. Fixed and periodic points in the probabilistic nomed and metric spaces, Chaos, Solitons \& Fractals 2006:28:1181-7.

[7] He Ji-Huan. El Naschie MS. Transfinite physics. Beijing: China Education and Culture Publising Co.: 2006.

[8] Lafuerza-Guillen B, Rodrigucz-Lallena J. Sempi C. Study ol boundedness in probabilistic normed spaces. J Math Anal Appl 1999:232:183-46.

[9] Lafuerza-Guillen B, Rodriguez-Lallena J. Sempi C. Probabilistic normed for linear operators. J Math Anal Appl 1998:220:462-76.

[10] Meneger K. Statistical metries. Proc Nat Acad of Sci USA 1942:28-535-7.

[11] Prochaska BJ. On randonm normed spaces. Pl, D Thesis, Clemson Univesi1y, 1967.

[12] Schweizer B. Sklar A. Probabilistic metric spaces. New-York. Amesterdam, Oxtord: North Holand: 1983.

[13] Schweizer B, Sklar A. Statistical metric spaces. Pacific J Math 1960;10:313-34.

[14] Šerstnev AN, On the notion of a random normed space. Dokl Akad Nauk SSSR 1963:149:280-3. 